\title{
Knowledge about the predictive value of reward conditioned stimuli modulates their interference with cognitive processes
}

\author{
Mateo Leganes-Fonteneau, ${ }^{1,2}$ Kyriaki Nikolaou, ${ }^{1,2}$ Ryan Scott, ${ }^{1,3}$ and Theodora Duka ${ }^{1,2}$ \\ ${ }^{1}$ School of Psychology, University of Sussex, Brighton BN1 9QH, United Kingdom; ${ }^{2}$ Sussex Addiction Research and Intervention Centre \\ (SARIC), University of Sussex BN1 9QH, United Kingdom; ${ }^{3}$ Sackler Centre for Consciousness Science, University of Sussex BN1 9QH, \\ United Kingdom
}

\begin{abstract}
Stimuli conditioned with a substance can generate drug-approach behaviors due to their acquired motivational properties. According to implicit theories of addiction, these stimuli can decrease cognitive control automatically. The present study ( $n$ $=49$ ) examined whether reward-associated stimuli can interfere with cognitive processes in the absence of knowledge about stimulus-outcome contingencies. Conditioned stimuli (CS) were paired with high-reward (HR) or low-reward (LR) probabilities of monetary reward using a Pavlovian learning task. Participants were categorized as Aware or Unaware of contingencies using a Bayesian analysis. CS were then used as task-irrelevant distractors in modified flanker and $N$-back tasks. Results show HR CS can generate increased interference in the flanker task for participants Unaware of contingencies, contributing further evidence for the existence of implicit Pavlovian conditioning. For the $\mathrm{N}$-back task, working memory performance was affected by HR CS, albeit only for Aware participants. These results suggest that CS can interfere implicitly with cognitive processes in a similar way to drug-related stimuli. Such an effect could occur in a stimulus-driven fashion, devoid of top-down goal-directedness. These findings have implications for the conceptualization and study of implicit processes in addiction and highlights the necessity to reconsider the measurement of such phenomena.
\end{abstract}

[Supplemental material is available for this article.]

Motivational properties of stimuli associated with substances are known to play a crucial role in the development of addictive behaviors. Through repeated associations with drug effects, drug-related stimuli acquire incentive salience (Goldstein and Volkow 2002; Berridge and Robinson 2003), a quality that ultimately drives and directs motivational responses. The instatement of those responses is posited to occur via mechanisms similar to that of Pavlovian reward conditioning (Stewart et al. 1984).

In humans, responsiveness to drug-related stimuli has been evaluated via attentional processes (Field and Cox 2008), and emotional (Pool et al. 2016) or autonomous (Carter and Tiffany 1999) reactivity. Attentional biases toward drug-related stimuli have been consistently observed for different substances (Bonson et al. 2002; Bradley et al. 2008; Moeller et al. 2009; Garland et al. 2012; Field et al. 2013), commonly evaluated using dot probe tasks (i.e., Townshend and Duka 2001) targeting overt attention allocation.

Other techniques assessing biases toward drug-related stimuli consist of eye-gaze measurements (Hogarth et al. 2006b) or interference tasks such as the Addiction-Stroop test (Cox et al. 2006). Interference by task-irrelevant drug-related stimuli has also been evaluated using working memory (WM) tasks (Hester and Garavan 2009) particularly with cocaine addicts.

Nikolaou et al. (2013a) further examined the mechanisms underlying cue interference in cognitive control using a modified flanker task (Eriksen and Schultz 1979); they found that task-irrelevant alcohol-related stimuli increased RT under high cognitive load, thus proving the effect of drug-related stimuli in

Corresponding authors: m.leganes-fonteneau@sussex.ac.uk; t.duka@ sussex.ac.uk

Article is online at http://www.learnmem.org/cgi/doi/10.1101/lm.048272.118. the attenuation of cognitive control resources necessary for correct task performance.

Others have also examined the ability of emotionally salient stimuli to interfere with $\mathrm{WM}$ using an $\mathrm{N}$-back task (Ladouceur et al. 2009). In this procedure, participants have to respond to targets occurring in a sequence of stimuli. In the case of a 0-back condition, this simply means responding when a given stimulus appears. In the case of an $\mathrm{N}$-back condition, a response is required when a stimulus is the same as one appearing $n$ stimuli earlier in the sequence. As the $n$ increases the cognitive load on WM also increases. Interference on performance resulting from the inclusion of drug-related nontarget stimuli in the sequence may result from a combination of explicit and implicit processes in drug addiction.

According to the dual process theory of addiction (Wiers and Stacy 2006), the cascade of events leading to drug-approach behaviors is supposed to occur implicitly, under the influence of stimuli associated with the substance (see also Tiffany 1990). Explicit motives activated in parallel support cognitive control mechanisms and are meant to impede or limit such tendencies. This tradeoff between an implicit appetitive system triggered by drug-related stimuli and an explicit cognitive control system based on regulatory executive signals can explain results such as those obtained in the alcohol flanker task (Nikolaou et al. 2013b).

Most experiments have investigated this matter using stimuli explicitly associated with a substance (e.g., alcohol bottles or

(C) 2019 Leganes-Fonteneau et al. This article is distributed exclusively by Cold Spring Harbor Laboratory Press for the first 12 months after the full-issue publication date (see http://learnmem.cshlp.org/site/misc/terms.xhtml). After 12 months, it is available under a Creative Commons License (AttributionNonCommercial 4.0 International), as described at http://creativecommons. org/licenses/by-nc/4.0/. 
cigarettes) for which the stimulus-outcome contingency is clearly defined due to their own nature, thus impeding an accurate exploration of implicit components of drug addiction. Even though the procedures themselves, based on task-irrelevant distractor effects, are thought to be implicit, the explicit attributes of drug-related stimuli generated through prior consumption experiences (Wiers et al. 2002) may affect task outcomes (Leganes-Fonteneau et al. 2018). Stimuli conditioned with nondrug rewards can generate value-driven responses equivalent to those of drug-related stimuli (Anderson et al. 2011), both attentional (Hogarth et al. 2006a; Jeffs and Duka 2017) and emotional (i.e., Austin and Duka 2010). As detailed by Anderson (2016), reward CS can, in a similar way as drug-related cues, override the interference of cognitive goals (e.g., Le Pelley et al. 2015), drive approach behaviors (e.g., San Martín et al. 2016), resist extinction procedures (e.g., Anderson and Yantis 2013), and trigger dopaminergic responses (Anderson et al. 2016b). This enables the use of reward learning procedures as an "in-vitro" model of addiction in laboratory settings.

However, in seeking to investigate the mechanisms underlying responsiveness toward reward-related stimuli, a key question is whether those responses can occur without conscious awareness (CA) of outcome contingencies, that is, in the absence of predictive knowledge about associations between conditioned stimuli (CS) and rewards. The ability of implicit reward-CS associations to produce hedonic and attentional responses has generated an extensive discussion (Lovibond and Shanks 2002), with research showing inconsistent results. Recent findings (Le Pelley et al. 2017), as well as previous research (i.e., Hogarth et al. 2005, 2006a), appear to show that CA is necessary for the development of responses in Pavlovian appetitive conditioning. These results may stem from the inadequacy of procedures used to assess learning, both in the measurement of conditioned responses (De Houwer 2006) and CA (Lovibond and Shanks 2002).

In a recent series of experiments, in which CA was carefully measured using a novel Bayesian approach (Dienes 2015; Sand and Nilsson 2016), we found that targets, paired with increased probabilities of monetary reward, gathered preferential attention in an Emotional Attentional Blink task (Leganes-Fonteneau et al. 2018). Importantly, this was observed in participants Unaware of stimulus-reward contingencies. These results are in line with the postulate that reward predictive stimuli can modulate attentional processes (Failing and Theeuwes 2017) even in the absence of CA.

As discussed above, research in the field of drug addiction has demonstrated preferential attentional responses toward task-irrelevant reward-related stimuli; made apparent by their influence on cognitive processes (Hester and Garavan 2009; Nikolaou et al. 2013b). Similar results have been obtained using secondary reinforcers. For example, using a modified color-naming Stroop task in which certain task-relevant colors (e.g., a blue font) were associated with monetary outcomes (Krebs et al. 2010), it was found that those colors facilitated task performance. Interestingly, the incentive value of rewarded colors subsequently transferred to task-irrelevant words associated with rewarded stimuli (e.g., the word "blue"), this time generating increased interference. This was considered an example of implicit reward learning. However, in this case, as in others (i.e., Anderson 2015), the implicit nature of the effect generated by CS can only be postulated as no stringent measures of CA were implemented. Anderson et al. (2012, 2016a) also reported the effect of CS as distractors on a modified flanker task, but again without examining the implicit aspects of learning.

Dual process theories of addiction (Wiers and Stacy 2006) indicate that the chain of events leading to appetitive behaviors is based on implicit processes triggered by CS. It is not clear however if stimuli implicitly associated with a drug, without conscious knowledge of outcome contingencies, can set off drug-approach behaviors (Hogarth et al. 2006a).
To help clarify this matter we investigated whether CS in the absence of CA can generate task-irrelevant interferences in cognitive control. As in previous research high reward (HR) CS generated preferential attentional responses in Unaware participants (Leganes-Fonteneau et al. 2018), we expect here to replicate those findings and that HR stimuli will interfere with inhibitory control to a greater extent than low reward (LR), again in the absence of CA. This would allow us to draw a parallel between the ability of implicitly CS to affect executive control (Anderson 2016) and the interference of drug cues on inhibitory mechanisms (Wiers and Stacy 2006).

To make sure that Pavlovian associations would occur in the absence of awareness we utilized a task-irrelevant reward learning procedure (Yokoyama et al. 2015), pairing stimuli belonging to two different categories with HR or LR probabilities of monetary reward. Using task-irrelevant procedures, it is possible to direct the focus of attention away from the stimulus-reward outcome, and in this way, delay explicit learning of stimulus-reward associations. We measured CA and metacognitive knowledge about contingencies on a trial-by-trial basis (Leganes-Fonteneau et al. 2018) to determine the explicit knowledge about outcome contingencies gained by participants. Using a Bayesian analysis for this purpose, we were able to gather sensitive evidence for the existence of nonconscious learning. Finally, emotional responses toward CS were measured, and the interference of CS on cognitive processes was assessed using modified flanker and $\mathrm{N}$-back tasks with different degrees of cognitive load.

We hypothesized that CS would have an effect on performance for both the $\mathrm{N}$-back and flanker tasks depending on their value (HR vs. LR) and that the extent to which participants were Aware or Unaware of the contingencies would modulate that interference.

\section{Results}

\section{Questionnaires}

Groups were matched on all baseline indices (i.e., Barratt Impulsiveness Scale-BIS, Alcohol Use Disorder Identification Test-AUDIT, Alcohol Use Questionnaire-AUQ, Positive and Negative Affect Schedule-PANAS, and Bodily Perception Questionnaire-BPQ; $P>0.2$, in all cases) except for Reversed Digit Span (see Table 1). Groups also did not differ in the distribution of male and female participants, $\chi^{2}(1, N=36)=1.446, P=0.229$ (ratio of female/total: $8 / 20$ for Unaware and 10/16 for Aware).

\section{Pleasantness}

There was a main effect of stimulus-type, $F_{(1,34)}=10.015, P=0.003$, reflecting, irrespective of $\mathrm{CA}$, increased pleasantness ratings toward HR $($ mean $=55.29, \mathrm{SD}=0.16)$ compared to LR CS $($ mean $=$ 43.97, $\mathrm{SD}=0.17)$.

This main effect was quantified by a significant CA by stimulus-type interaction, $F_{(1,34)}=7.899, P=0.008$. Thus, Aware participants rated HR CS as being more pleasant than LR CS, $t$ $(15)=3.182, P=0.006, B_{U(0,0.06)}=3.3998$. In contrast, there was no sensitive difference in pleasantness ratings between HR and LR CS in the Unaware group, $t(19)=0.362, P=0.721, B_{U(0,0.06)}=$ 0.9054 (see Fig. 1).

\section{$N$-back}

\section{Accuracy $\mathrm{N}$-back}

For Aware participants, the analysis of the percentage of correct responses showed a main effect of load, $F_{(1,45)}=29.307, P<0.001$, with participants performing less accurately in 2-back blocks (mean $=79.43, \mathrm{SD}=8.22)$ than in 0-back blocks $($ mean $=90.10$, 
Table 1. Results and descriptives comparing questionnaires and demographic scores between contingency Aware and Unaware participants

\begin{tabular}{|c|c|c|c|c|c|c|}
\hline & Contingency Aware & $n=16$ & Contingency Unaware & & $n=20$ & \\
\hline & \multicolumn{6}{|c|}{ Between-group comparison } \\
\hline & Mean & SD & Mean & SD & $t(34)$ & $P$ \\
\hline Age & 20.13 & 5.39 & 19.55 & 1.23 & 0.165 & 0.870 \\
\hline BIS-11 & 63.81 & 8.20 & 64.2 & 5.87 & 1.136 & 0.264 \\
\hline AUDIT & 7.69 & 4.70 & 7.8 & 5.52 & 0.886 & 0.382 \\
\hline Binge score & 25.69 & 16.45 & 19.29 & 17.06 & 0.486 & 0.630 \\
\hline AUQ̊ score & 41.43 & 25.53 & 33.5 & 27.51 & 0.983 & 0.333 \\
\hline PANAS Positive & 0.47 & 0.15 & 0.44 & 0.20 & 0.165 & 0.870 \\
\hline \multirow[t]{2}{*}{ Porges } & 2.52 & 0.50 & 2.31 & 0.75 & 1.136 & 0.264 \\
\hline & Mean & SD & Mean & SD & $Z$ & $P$ \\
\hline Reverse Digit & 3.21 & 0.97 & 4.13 & 1.26 & 2.903 & 0.043 \\
\hline PANAS Negative & 0.10 & 0.07 & 0.12 & 0.11 & 0.161 & 0.888 \\
\hline
\end{tabular}

$\mathrm{SD}=7.89$ ). Similarly, Unaware participants also had lower accuracy scores in the 2 -back (mean $=84.65, \mathrm{SD}=7.95)$ than the 0 -back condition $\left(\right.$ mean $=88.49, \mathrm{SD}=10.83$ ); main effect of load, $F_{(1,54)}=$ $8.006, P=0.007$. There were no other significant main effects or interactions neither for Aware nor for Unaware participants when looking at the percentage of correct responses, $F \mathrm{~S}<0.427, P>0.516$.

Analyses of net differences, resulted in no significant main effects or interactions neither for Aware, nor for Unaware participants, $F \mathrm{~s}<0.935$, ps $>0.338$.

For proportion of correct Hits, there were no significant interactions or main effects, neither for Aware, nor for Unaware participants, $F s<1.638$, ps $>0.207$.

\section{Latencies $\mathrm{N}$-back}

With respect to latencies taking into account all trials, Aware participants were overall slower on 2-back trials (mean $=2.76, \mathrm{SD}=$ 0.13 ) compared to 0-back trials (mean $=2.74, \mathrm{SD}=0.10$ ); main effect of load, $F_{(1,15)}=5.624, P=0.032$. There were no other significant main effects or interactions in the Aware group, $F \mathrm{~s}<2.597$, $p s>0.128$. The analysis performed in the Unaware group did not result in any statistically significant main effect or interaction, Fs $<1.015$, ps $>0.327$.

With respect to latencies on target trials only: the analysis in the Aware group showed a marginally significant main effect of stimulus-type, $F_{(1,15)}=3.367, P=0.086$, indicating that latencies to LR targets were faster (mean $=2.70, \mathrm{SD}=0.07$ ) than to HR targets (mean $=2.72, \mathrm{SD}=0.07)$. In addition, there was a marginally significant stimulus-type by load interaction, $F_{(1,15)}=3.875, P=$ 0.068 . Post hoc paired-samples $t$-tests showed that responses on HR target trials were marginally slower than responses on LR target trials in the 2-back block, $t(15)=2.122, P=0.051$ (see Fig. 2 ). No other simple effect was statistically significant, $P>0.149$. Finally, the main effect of load was not statistically significant, $F_{(1,15)}=$ $0.587, P=0.456$.

For Unaware participants, there were no statistically significant main effects or interactions, $F<1.733, P>0.205$, in all cases.

\section{Flanker task}

\section{Accuracy flanker}

For Unaware participants there was a main effect of stimulus-type on accuracy, $F_{(2,85)}=6.834, P=0.002$, explained by significantly lower accuracy overall on $\mathrm{HR}$ (mean $=94.90, \mathrm{SD}=3.84)$ compared to Control trials (mean=97.07, SD=2.28), $Z=3.675, P<0.001$, and marginally lower accuracy on $\mathrm{LR}($ mean $=95.68, \mathrm{SD}=3.84$ ) than Control trials, $Z=1.945, P=0.052$. There was no significant difference in accuracy between HR and LR trials, $Z=0.857, P=$ 0.391 . The main effect of congruency was also significant, $F_{(1,85)}$ $=152.970, P<0.001$, and reflected increased accuracy in general on congruent (mean $=99.38, \mathrm{SD}=1.05)$ than incongruent trials (mean $=92.39, \mathrm{SD}=5.03$ ). Both of these main effects were quantified by an interaction between stimulus-type and congruency in the Unaware group, $F_{(2,85)}=6.420, P=0.002$, due to accuracy being higher for Control than HR stimuli across levels of congruency, $\chi^{2}(1)=12.807, P=0.001$. Wilcoxon post hoc tests showed a significant difference between Control and HR trials in the incongruent condition, $Z=2.255, P=0.024$, that did not occur in the congruent condition, $Z=.447, P=0.655$ (see Table 2).

Similarly, for Aware participants, there was also a main effect of stimulus-type on accuracy, $F_{(2,70)}=7.820, P<0.001$, explained by overall lower accuracy for HR $($ mean $=93.43, \mathrm{SD}=5.39)$ compared to Control trials (mean $=95.37, \mathrm{SD}=5.98$ ), $Z=3.819, P<$ 0.001 , and lower accuracy on LR (mean $=93.06, S D=7.48)$ compared to Control trials, $Z=2.111, P=0.035$. There was no difference in accuracy between $\mathrm{HR}$ and LR trials, $Z=0.175, P=0.861$.

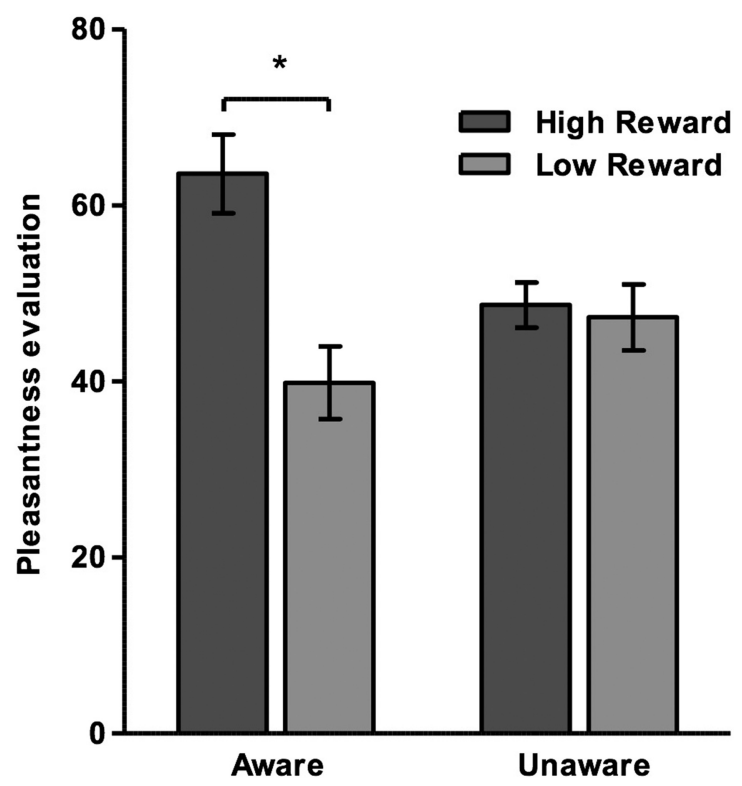

Figure 1. Pleasantness toward conditioned stimuli. Pleasantness ratings toward high-reward and low-reward stimuli depending on contingency awareness. *Only Aware participants developed preferential emotional responses toward high-reward stimuli, $t(15)=3.182, P=0.006$. 


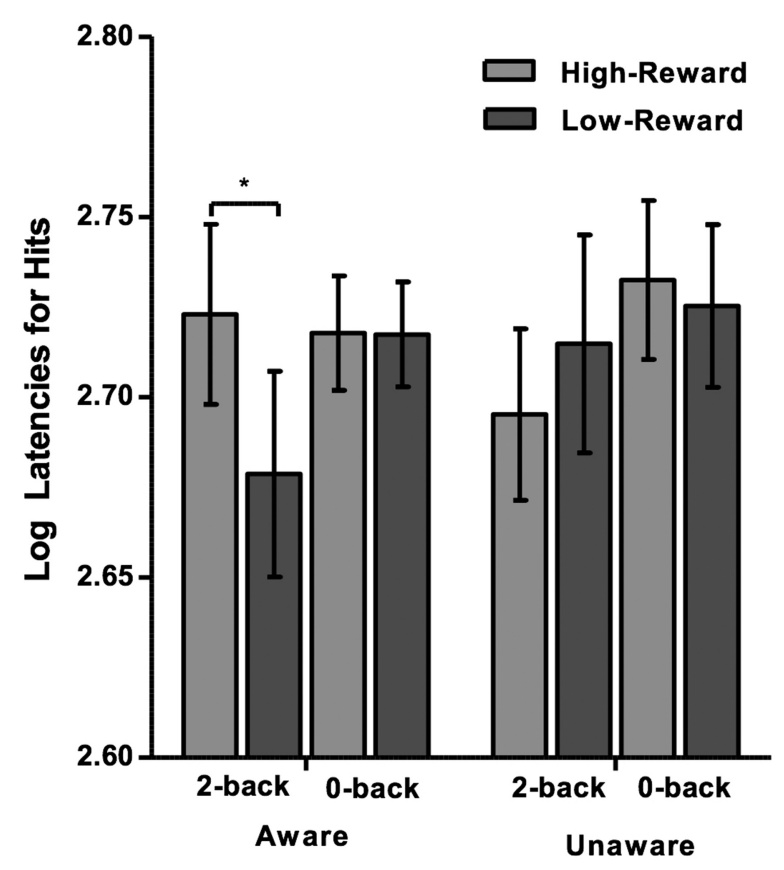

Figure 2. Reaction times during the $N$-back task between Aware and Unaware participants for Hits. *For Aware participants Latencies in the 2-back condition toward high reward were higher than those toward low reward, $P=0.051$.

The main effect of congruency was also significant, $F_{(1,70)}=$ $146.051, P<0.001$, and reflected increased accuracy in general on congruent (mean $=99.14, \mathrm{SD}=1.13$ ) than incongruent trials (mean $=88.77, \mathrm{SD}=11.08$ ). There was however no interaction between stimulus-type and congruency, $F_{(2,70)}=2.360, P=0.102$.

\section{Flanker effect}

Unaware participants showed a main effect of stimulus-type, $F_{(2,34)}$ $=6.506, P=0.004$. HR CS generated more interference than both LR, $P=0.042$, or Control stimuli, $P=0.006$ (see Fig. 3 ).

This was not observed, in Aware participants, $F_{(2,28)}=0.606$, $P=0.553$.

\section{Differences between groups}

Out of the three-way bootstrapping procedure, we found that the flanker effect was significantly lower in Unaware compared to Aware participants for LR, 95\% CI [-39.25 to -2.27], and Control stimuli, 95\% CI [-40.29 to -7.67], but not for HR stimuli, 95\% CI [ -15.89 to 17.05$]$. This highlights the differences between Aware and Unaware participants in the interference generated by CS. See Supplementary Materials for complete results of the bootstrapped comparisons of between-group effects.

\section{Discussion}

The present study set out to examine the extent to which reward CS can interfere with cognitive processing and the degree to which this remains true in the absence of CA. By using a task-irrelevant Pavlovian conditioning paradigm and setting stringent criteria in the determination of CA, it was possible to reliably identify implicit influences. The use of a Bayesian approach provides a sensitive measurement of the unconscious nature of learning, countering otherwise well-grounded methodological criticisms regarding measurements of implicit learning (Lovibond and Shanks 2002), due to lax determinations of unconscious processes (Shanks 2016).

We observed significant differences between groups in response latencies generated by CS. The flanker effect was higher for HR CS compared to LR or Control, albeit only in participants Unaware of the contingencies. On the other hand, in the $N$-back task, under high cognitive load, a higher interference in the presence of HR CS compared to LR CS was only found in Aware participants. Subjective hedonic responses to HR CS, as measured by pleasantness ratings, were also only seen in participants Aware of contingencies; Unaware participants displayed an insensitive response pattern.

The development of hedonic responses congruent with reward signaling only in Aware participants clarifies recent findings (Leganes-Fonteneau et al. 2018) suggesting that CA is necessary for the development of subjective emotional responses. Previous research from our own group had found instances of hedonic responses in the absence of CA (Jeffs and Duka 2017). These incongruent results may again be due to the use of less stringent CA categorizations. However, insensitive results, with regard to pleasantness for Unaware participants (as shown with Bayesian analyses), indicate that we cannot fully discard the development of such responses also in this group. Other factors contributing to the development of hedonic conditioned responses, such as interoceptive abilities (Pollatos and Schandry 2008), may ultimately help resolve such disparities. However, it seems clear that the assessment of subjective hedonic responses is by no means an adequate tool for the examination of implicit learning (De Houwer 2006). Finally, demand characteristics may explain why Aware participants rate HR stimuli as more pleasant, not because of an acquired incentive value, but as a need to comply with the awareness of contingencies.

As expected, decreases in performance (accuracy and latencies) were found for the flanker task, in the incongruent compared to the congruent condition; also, as expected in the $\mathrm{N}$-back task, accuracy decreased in the 2-back compared to the 0-back blocks, showing the suitability of the procedures to generate increased cognitive load.

Results of the flanker task are in line with data recently obtained by our group (Leganes-Fonteneau et al. 2018) and by others (i.e., Bourgeois et al. 2016) demonstrating the ability of reward CS to grab preferential attention implicitly. This interference is also congruent with previous examples of reward conditioning effects on a flanker task (Anderson et al. 2012, 2016a). HR CS, paired with increased probabilities of reward, generated more cognitive

Table 2. Accuracy during the flanker task. Accuracy during the flanker task depending on contingency awareness, cognitive load, and stimulus-type

\begin{tabular}{|c|c|c|c|c|c|c|c|c|c|c|c|c|}
\hline & \multicolumn{6}{|c|}{ Congruent } & \multicolumn{6}{|c|}{ Incongruent } \\
\hline & \multicolumn{2}{|c|}{ High reward } & \multicolumn{2}{|c|}{ Low reward } & \multicolumn{2}{|c|}{ Control } & \multicolumn{2}{|c|}{ High reward } & \multicolumn{2}{|c|}{ Low reward } & \multicolumn{2}{|c|}{ Control } \\
\hline & Mean & SD & Mean & SD & Mean & SD & Mean & SD & Mean & SD & Mean & SD \\
\hline Unaware & 99.54 & 1.07 & 98.92 & 2.88 & 99.69 & 0.90 & 90.28 & 7.70 & 92.44 & 5.94 & 94.44 & 4.57 \\
\hline Aware & 99.07 & 1.36 & 98.52 & 2.54 & 99.81 & 0.72 & 87.78 & 10.85 & 87.59 & 13.36 & 90.93 & 11.38 \\
\hline
\end{tabular}




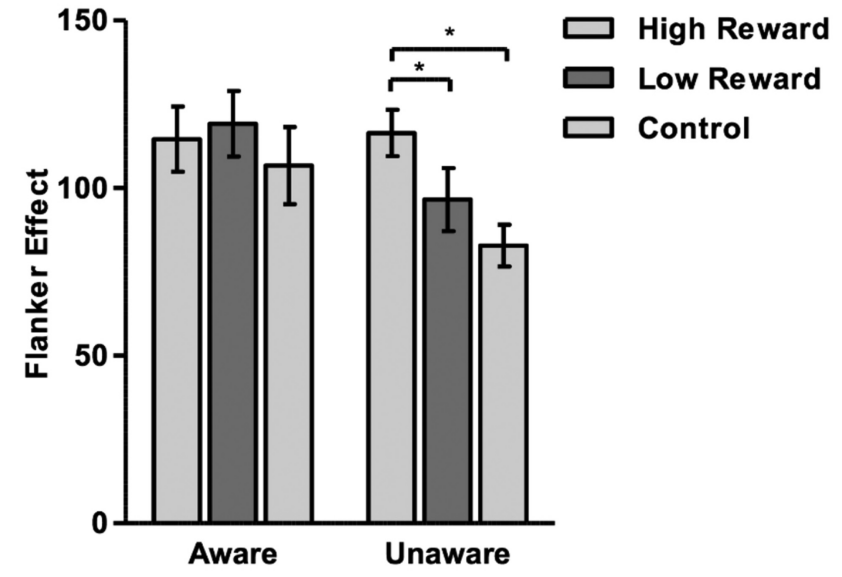

Figure 3. Flanker scores. Latency difference scores for congruent versus incongruent trials in the flanker task by contingency awareness and stimulus-type. *For Unaware participants, high cognitive load generates more interference in HR trials compared to $L R, t(17)=2.740, P=0.014$, and control stimuli, $t(17)=3.615, P=0.002$.

interference compared to LR CS. Importantly that happened solely for participants Unaware of contingencies. These results are relevant enough by themselves as they provide further evidence for the existence of implicit Pavlovian conditioning effects.

Research findings relating to the nature of the attentional processes underlying task interference by irrelevant distractors have proven to be inconsistent. On one hand, attentional resources are posited to be necessary for task interference (Pessoa and Ungerleider 2004) countering the argument of automaticity of salience effects and converging with the interpretation of attentional biases toward drug cues acting as a top-down albeit involuntary mechanism (Brown et al. 2018). However, value-driven attentional capture (Anderson et al. 2011) is also posited to occur involuntarily. In our case, as the interference generated by HR CS occurs without CA of their reward predictive value, we can posit that their influence is implicit, devoid of goal-directedness.

The ability of CS to interfere with cognitive control tasks in a stimulus-driven fashion has twofold implications for implicit theories of addiction. On the one hand, this mere effect shows that stimuli associated with a reward can generate approach behaviors in a nondeclarative or explicit way and can interfere with cognitive control mechanisms. Considering the similarities between experimental models of reward learning and the attentional correlates of addiction (Anderson 2016) our results provide further evidence for the relevance of implicit processes in substance use disorders (Wiers and Stacy 2006). This implies a need to reconsider how cognitive control interference induced by drug-related stimuli implicitly can be tested. Explicit drug cues typically utilized in relevant experiments (e.g., pictures of alcohol bottles for alcohol-related cognitive bias) might not allow implicit mechanisms involved in cognitive biases to be revealed. On the other hand, if we consider that the effects of reward-associated cues can occur on cognitive interference implicitly, as shown here, we can extrapolate and add to our understanding of mechanisms underlying drug-cue interference in cognitive control (i.e., on an equivalent flanker task Nikolaou et al. 2013b), that drug-cue interference could be affected, at least partially, via implicit and involuntary processes detached from goal-directedness (Hester and Garavan 2009).

It is puzzling that Aware participants did not show a stronger flanker effect of HR CS compared to LR CS compared to Unaware participants. This might be explained by an overall decrease in baseline performance for Aware participants (illustrated by higher flanker effect for Control trials in Aware compared to Unaware participants, $\left(t_{(31)}=2.302, P=0.028\right)$. Such an effect could impede an appropriate interference by HR CS. Another possibility is that due to the conscious knowledge about contingencies, participants were consciously trying to identify the HR CS (exploiting attentional resources to achieve this), in order to minimize their interference. Such a cognitive process could explain the high flanker effect seen also in Control trials for that group.

On the $\mathrm{N}$-back task, stimulus interference was observed only for Aware participants. Under high cognitive load (2-back condition), latencies for Hits in the presence of HR CS were longer than toward LR CS. We can assume that this effect is due to the increased salience of HR CS.

It seems therefore that cognitive mechanisms underlying approach to reward-related stimuli tested by different tasks are influenced differently by CA. CS effects in the flanker task can be explained according to bias competition models (Desimone and Duncan 1995) by which under limited attentional resources (such as high cognitive load) salient stimuli will grab attention and interfere with the task (Vuilleumier 2005), in our case implicitly. Regarding the specific mechanisms by which the interference of HR CS occurs in the flanker task, we can draw several hypotheses. One possibility is that HR stimuli broaden the focus of attention toward the stimulus compound, and as such more attention is allocated as well to the distracting arrows, increasing their interference with cognitive control. Another possibility is that attention is directed toward HR stimuli and away from the main task, impacting task performance. Finally, HR stimuli may have a broad effect on cognitive control, impairing the ability to inhibit the interference of incongruent flankers. Further understanding the mechanisms by which implicitly CS exert their interference in cognitive control might also help understand how other reward-related stimuli (i.e., drug cues) generate such effects. ${ }^{4}$

We need however to explain why no effects were found in the $N$-back task for Unaware participants. The $N$-back task differs from the flanker task as it requires less attentional demand (detecting a color vs. a target arrow embedded in distractors) but involves more complex cognitive processes (recalling the color of a previous image to respond, while at the same time ignoring target irrelevant information vs. overcoming distractors). Previous research found that masked presentations of emotional distractors altered $N$-back performance, albeit not for high load trials (Uher et al. 2014). It is possible that interference on the $N$-back task does not directly target attentional mechanisms but rather WM and inhibitory processes (Kensinger and Corkin 2003), and that a more conscious presentation of distractors, or of their incentive value, is necessary for interference to occur in WM (LeDoux 2002). It is possible as well that on the $N$-back task it was easier to overcome the effect of CS as distractors by focusing on the color of the frame, whereas on the flanker task CS appeared underneath the relevant arrows. This may explain the lack of clearer results using this task overall.

The results obtained in this study are subject to a number of limitations. The effects of HR CS on response latencies in the $\mathrm{N}$-back task are observed solely on accurate target trials, that is, on relatively few instances, maybe because HR CS only affect trials in which the colored frame matches the 2-back stimulus, interfering with target recognition.

The observation that interference in the flanker task was not found for Aware participants remains to be fully explained. While it may be possible to provide an account of these results based on the effect of conscious knowledge about contingencies,

${ }^{4}$ We thank one of the reviewers for suggesting these mechanisms. 
it would be informative to compare the effect of emotional stimuli or masked distractors to the interference generated by CS on both tasks (Nikolaou et al. 2013a).

A final limitation of this study is the seemingly underpowered sample. This is partially due to the use of a Bayesian categorization which implies a proportion of the sample will be discarded due to their insensitive results on contingency awareness. As much as this constitutes a limitation of the methodology, the need to improve the way implicit cognitions are represented (Dienes 2015) justifies it. Further studies on implicit conditioning should aim at clarifying our results, particularly the $N$-back task, by increasing the sample size. The results on the flanker task seem however to provide sufficient evidence for the existence of implicit learning effects. Additionally, we have limited the comparison between Aware and Unaware participants to the categorization obtained using Type I scores. Type II scores were vastly insensitive in this study, precluding their use as an effective categorization tool. Still, the role of metacognitive CA in the development of learning deserves future explorations.

In conclusion, implicit processes play a crucial role in the development of drug addiction, particularly in drug-approach behaviors. We observed a clear interference in cognitive control by stimuli conditioned with reward in the absence of CA. This effect provides further evidence for the existence of implicit Pavlovian conditioning and has implications for the understanding of dual process theories of addiction. Uncovering implicit mechanisms of drug-approach behaviors may prove essential for the development of novel treatments in substance use disorders.

\section{Materials and Methods}

\section{Participants}

Forty-nine Psychology students from the University of Sussex completed the experiment (mean age $=20.04, \mathrm{SD}=3.34 ; 25$ females). Exclusion criteria were a history of mental disease and undergoing heavy medical treatment at the time of the study. All participants were given course credits and $£ 2$ for taking part in the study, and the study was approved by the University of Sussex Life Sciences Ethics Committee.

\section{Measures}

\section{Questionnaires}

Reversed Digit Span measurements were used to index WM capacity (Wechsler 2008; Redick and Lindsey 2013).

The AUQ (Mehrabian and Russell 1978) was used to assess average weekly alcohol use over the past 6 mo. The questionnaire also provides a binge drinking score based on the speed of alcohol consumption, and the number as well as the proportion of times that participants were drunk in the last 6 mo (Townshend and Duka 2002).

Severity of alcohol use was measured with the 10-item AUDIT (Saunders et al. 1993). Alcohol scores were measured as an exploration of the relationship between addictive disorders and aberrant reward processing (Balodis and Potenza 2015).

The PANAS (Watson et al. 1988) was used to measure positive and negative mood at the start of the study session. Participants rate how they feel at that moment using a 5-point Likert scale $(1=$ "very slightly," $5=$ "extremely"). The questionnaire consists of 10 items per construct.

The Body Perception Questionnaire (BPQ; Porges 1993) is a 45-item questionnaire evaluating the subjective ability to detect internal bodily sensations. Participants had to indicate on a 5 -point Likert scale $(1=$ "never," $5=$ "always") the frequency with which they felt different sensations, for example, facial twitches or bowel movements. This questionnaire, as well as other interoceptive measurements, was obtained in the context of a secondary analysis on the role interoceptive processing in reward learning, not detailed in this paper.

Finally, the BIS (Patton et al. 1995) is a 30-item questionnaire evaluating different factors contributing to overall impulsiveness, namely, Attentional, Motor, and Nonplanning impulsivity.

\section{Conditioning task}

A task-irrelevant conditioning procedure (Yokoyama et al. 2015; Leganes-Fonteneau et al. 2018) was implemented in order to train participants to associate high and low probabilities of monetary reward with two different categories of CS. Thirty-six geometrical stimuli belonging to each CS category (squares vs. octagons) were produced with Ink-Scape vector design software. ${ }^{5}$

On each trial, a stimulus from one CS category (i.e., a square or an octagon) was presented on the computer screen with an overlaid green- or yellow-colored square. Participants were asked to press a green or yellow key depending on the color of the square. Stimuli remained on screen for $2000 \mathrm{msec}$ or until a response was made. If the response was correct, participants could win 10p. For HR CS, the probability of winning was $90 \%$. For LR CS, the probability was $10 \%$. After a response was recorded feedback about the outcome of the trial was provided "You win 10p" or "You win nothing") for 1500 msec. Trial outcomes depended solely on the CS presented during the trial and the associated probabilities of reward. The stimulus category was counterbalanced across participants.

On $50 \%$ of the HR trials and on $50 \%$ of the LR trials, participants had to indicate if they thought they would win money (Yes/No expectancy responses). Following the expectancy response, they were also asked to rate how confident they were about their response on a 5-point Likert scale $(1=$ "completely guessing," $2=$ "more or less guessing," $3=$ "fairly sure," $4=$ "almost certain," $5=$ "completely certain"). The measurement of accuracy and confidence on reward prediction allowed us to determine participants' metacognitive knowledge about contingencies (Barrett et al. 2013).

The conditioning procedure comprised a total of five blocks, with 72 trials in each block. At the end of each block, participants transferred the amount earned coins from a bank box to their "earnings" box. At the end of the final block, they were told how much money they had won in total.

Participants were kept naïve about the contingencies between the CS categories and the reward outcome probabilities although they were told that they could win money at the end of each trial. They were also told that they would be asked a series of questions about their expectancy and confidence and that their responses to those questions would not affect the outcome of the trial in terms of reward probability.

\section{Pleasantness measurement}

Immediately after the conditioning procedure participants rated the pleasantness associated with each category of CS. Eighteen squares and 18 octagons (randomly selected from the original 72 stimuli) were presented in a random order, one at a time, and participants were asked to rate how pleasant they found each stimulus on a 5 -point Likert scale ( $1=$ not pleasant at all; $5=$ extremely pleasant).

\section{CS N-back task}

Each trial of the CS N-back task began with a fixation cross in the center of screen (jittered 1-3 sec; average $2 \mathrm{sec}$ ). This was followed by the stimulus display for $500 \mathrm{msec}$, followed by a response interval for 1000 msec.

The stimulus display consisted of a CS surrounded by a colored frame. On $50 \%$ of the trials, the CS was a HR CS, whereas

\footnotetext{
${ }^{5}$ See Leganes-Fonteneau et al. (2018) for a detailed description of stimulus development, Figures 4 and 5, for example, and Supplementary Material for the complete collection of stimuli: https://osf.io/t9qu6/?view_only= 242170271 cc1418aae7bb65f6c744f85.
} 
on the remainder $50 \%$ of the trials it was a LR CS. The color of the frame was either a primary color (i.e., red, blue, and yellow) or a nonprimary color (pink, orange, and green).

The task consisted of two conditions. In the 0-back condition, participants were instructed to press one button if the color of the frame was a primary color (target trial) and another button if it was a nonprimary color (control trial) as quickly and as accurately as possible. In the 2-back condition, participants had to remember the color of the frame and press one button if the color of the frame matched the one shown two trials before (target trial) and another button if the colors did not match (see Fig. 4).

A total of eight blocks (four 0-back and four 2-back) were presented in an ABAB order. A 0-back block was always presented first. At the start of each block, a short instructions screen (jittered duration $4-8 \mathrm{sec}$, average $6 \mathrm{sec}$ ) reminded participants what they should do in that block.

Each block consisted of eight control and four target trials. In half of the control trials and in half of the target trials, the image presented was a HR CS, with the other half being LR CS. In order to match the visual properties of each block, target trials always consisted of primary colors and control trials of nonprimary colors regardless of the $\mathrm{N}$-back condition. Within blocks, the colors surrounding HR and LR CS were also matched.

In each 2-back block, at least one (maximum two) lure trials were introduced. These trials were control trials in which the color of the frame matched the one presented 1-back or 1-forward. Lure trials were allocated equally often between HR and LR image conditions.

The percentage of correct responses was calculated for each $\mathrm{N}$-back and stimulus-type condition separately. We also computed the proportion of Hits (proportion of accurate Target trials), and net differences subtracting False Alarms rates (proportion of "target-like" responses on Control trials) from the proportion of Hits for each of the conditions. Reaction times to correct responses (i.e., latencies) were calculated for each $\mathrm{N}$-back and stimulus-type condition. Reaction times to correct target trials (i.e., latencies for Hits) were also computed separately for each $N$-back and stimulustype condition.

Finally, participants completed two practice blocks, one for each $N$-back condition, in which a plain gray background was presented as part of the stimulus display. A minimum accuracy of $65 \%$ in each practice block was required to proceed to the real task.
Participants not reaching this threshold were given the task instructions again and repeated the practice blocks.

\section{CS flanker task}

The task was adapted from Nikolaou and colleagues (2013a).

Each trial began with the presentation of a fixation cross for a jittered duration (850-1150 msec), followed by the stimulus display for $800 \mathrm{msec}$, and a response interval for $700 \mathrm{msec}$ (see Fig. 5).

The stimulus display consisted of a horizontal row of five arrows superimposed on either a plain gray background, or on task-unrelated background images that belonged to either the HR or the LR CS categories. The central arrow was the target, and was surrounded by two distracting arrows (flankers) on either side. Participants were instructed to ignore the flankers and press one key if the central arrow was pointing to the left, and another key if it was pointing to the right, as quickly and accurately as possible. In the congruent condition flankers pointed in the same direction as the target (e.g., $<<<<<$ ). In the incongruent condition flankers pointed in the opposite direction to the target (e.g., $>><>$ $>$, bold font added only for illustration). There were four different flanker and target combinations (target pointing left or right and flankers pointing left or right).

Each of the flanker combinations was superimposed on each of 20 selected HR and LR CS images, to generate $80 \mathrm{HR}$ and 80 LR trials (40 in the congruent and 40 in the incongruent condition). An additional 40 congruent and 40 incongruent trials with a plain gray background (i.e., control trials) were also included to generate a single task block of 240 trials in total. Trial order was pseudorandomized to avoid presentation of the same background and same congruency condition for more than three consecutive trials.

Mean latencies and accuracy scores (\% of correct responses) were computed for each background image (i.e., HR, LR, and Control) under both the congruent and incongruent conditions. The flanker effect was also computed for each image condition separately as the difference in average latency in the incongruent condition minus the average latency in the congruent condition.

Participants completed 40 practice trials, 20 congruent and 20 incongruent, with only the gray plain background before the main block. A minimum accuracy of $70 \%$ was required to proceed to the experimental block (achieved by all participants).

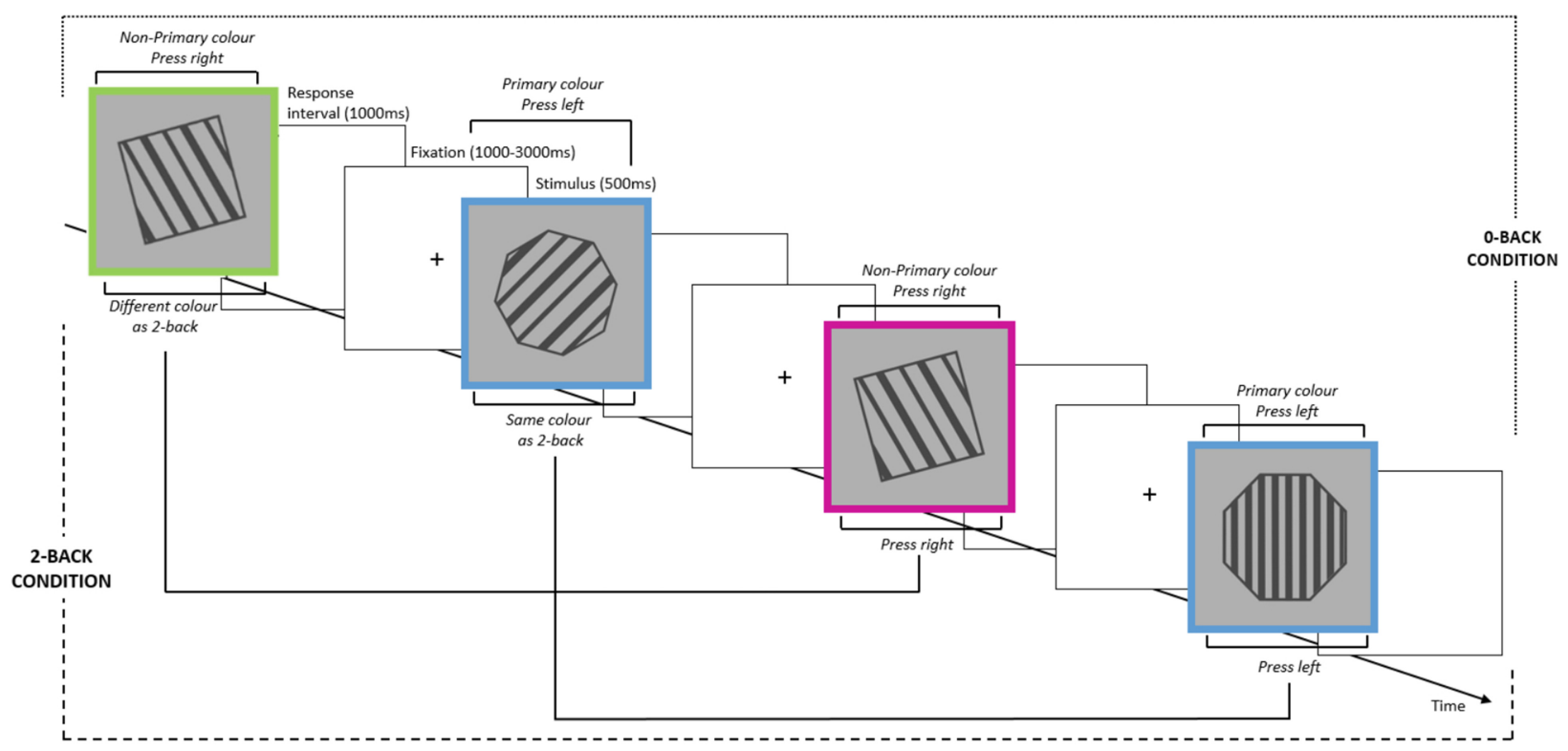

Figure 4. $\mathrm{N}$-back task. The task consisted of two different conditions. In the 0 -back condition, participants indicated whether the color of the frame was a primary or a nonprimary color. In the 2-back condition, they had to indicate whether the color of the frame matched the one presented two trials before. High- and low-reward CS were task-irrelevant. 


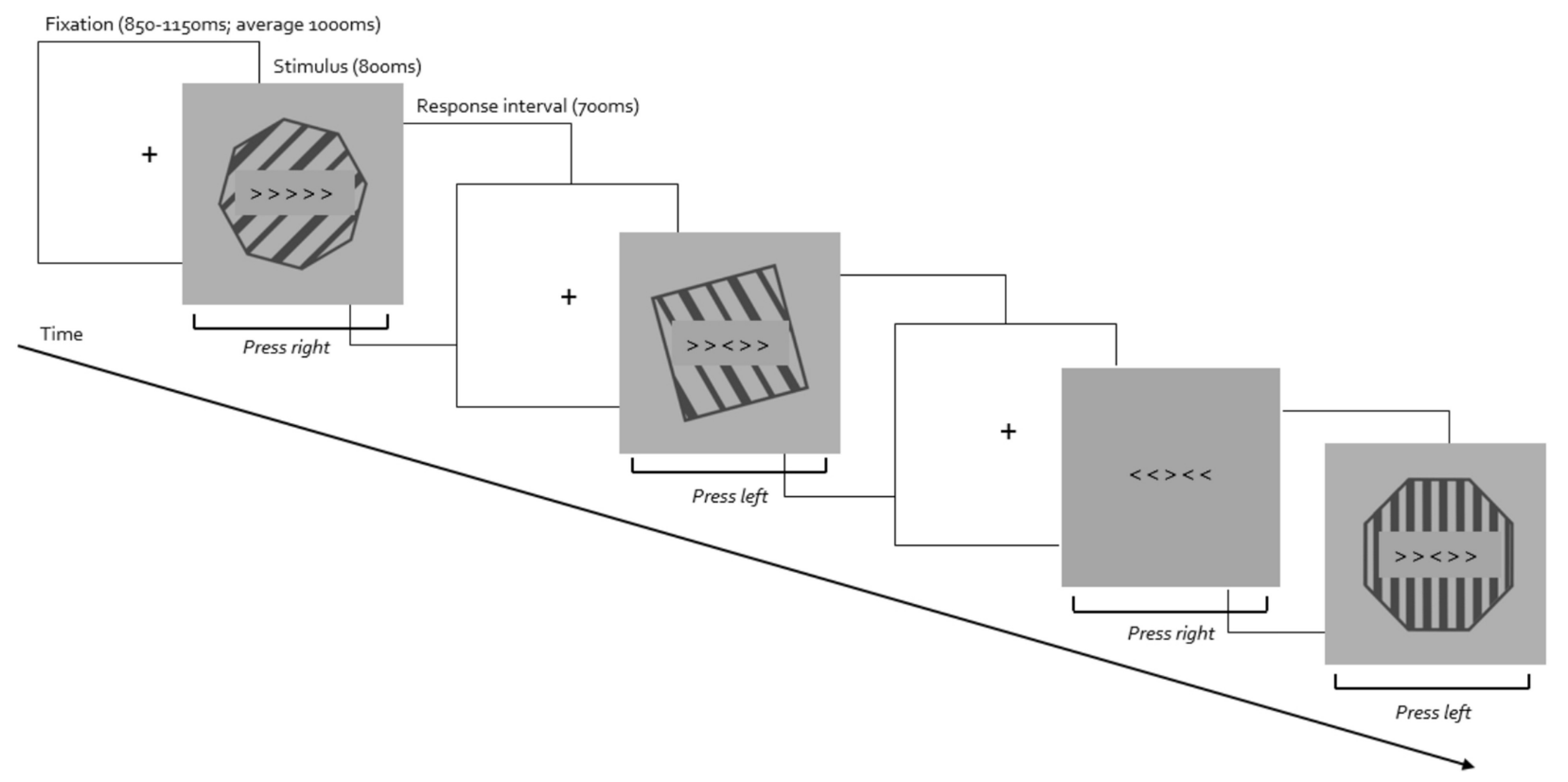

Figure 5. Flanker task. Participants responded depending on the direction of the central arrow, which could be surrounded by congruent or incongruent arrows. High- and low-reward conditioned stimuli as well as gray control backgrounds were task-irrelevant.

\section{Procedure}

Each participant completed a single testing session. They first gave written informed consent that they agreed to take part in the study. This was followed by the completion of the AUQ, AUDIT, PANAS, BIS-11, and BPQ. They then completed two tasks designed to measure interoceptive awareness (detailed in Garfinkel et al. (2015), see Supplemental Materials for data), followed by the reward conditioning procedure and pleasantness evaluation. Finally, they completed the CS $N$-back and flanker tasks in a counterbalanced order. At the end of the experiment, they were debriefed and compensated for their participation.

\section{Data analysis}

\section{Bayesian analysis}

Bayesian analyses provide a statistical tool with which the sensitivity of results can be determined. This way it is possible to extract conclusions from nonsignificant findings generated by frequentist statistical approaches (Dienes 2014). A Bayes factor (B) above 3 shows compelling evidence toward the alternative hypothesis (i.e., two means are different), whereas a $B$ below $1 / 3$ provides substantial evidence toward the null hypothesis (i.e., there is no difference between two means). A $B$ between 3 and 1/3 implies there is not enough evidence in either direction.

Bayes factors were used (a) as a tool for sensitive categorization of participants into those who were aware of the CS-HR/LR contingencies and those who were not (see Leganes-Fonteneau et al. 2018 for details and below for a summary) and (b) to examine the sensitivity of within group comparisons of the pleasantness ratings (see "Pleasantness" below).

\section{Contingency awareness categorization}

Determining the unconscious nature of a mental process requires evidence of an inability or failure to consciously perceive that process. This typically involves asserting the null hypothesis that performance on some knowledge-related task is no different to chance, which cannot be evaluated using traditional frequentist statistical analyses (Dienes 2015). Therefore, a Bayesian approach (Sand and Nilsson 2016) was used to categorize participants as
Aware or Unaware of contingencies using the data gathered from the conditioning procedure (Leganes-Fonteneau et al. 2018).

Using Signal Detection Theory methods (Stanislaw and Todorov 1999; Barrett et al. 2013), we computed the number of Type I: Hits (responding "Yes" on a HR trial), Correct Rejections (responding "No" on a LR trial), False Alarms (responding "Yes" on a LR trial), and Misses (responding "No" on a HR trial) from blocks 4 and 5 of the conditioning task. Type I scores reflect performance accuracy.

Type II scores provide an account of the metacognitive knowledge generated during the task using the relationship between accuracy and confidence responses (Kunimoto et al. 2001). Confidence responses were transformed to a dichotomous variable: responses of two or below (i.e., "more or less guessing" or "completely guessing") were classified as "not confident," and those above two as "highly confident." Thus, a Type II Hit was defined as a Type I Hit or Correct Rejection with high confidence; a Correct Rejection as a Type I Miss or False Alarm with low confidence; a False Alarm as a Type I Miss or False Alarm with high confidence; and a Miss as a Type I Hit or Correct Rejection with low confidence.

Logistic d1 $1^{\prime}\left(\operatorname{Logd} 1^{\prime}\right)$ and Standard Error $\mathrm{d} 1^{\prime}$ (SEd1') scores were calculated for each participant as well as Logd2' and Sed2' scores. As $\mathrm{d} 2^{\prime}$ scores rarely exceed $\mathrm{d} 1^{\prime}$ scores (Dienes 2015), a Bayes factor was calculated for each participant on their Logd2' modeling $\mathrm{H}_{1}$ with a Uniform going from 0 (chance level) to their own Logd $1^{\prime}$ as a prior. Participants with $B<1 / 3$ were categorized as Metacognitively Unaware, whereas those with a $B>3$ were considered Metacognitively Aware, and the rest as insensitive.

The mean Logd1' score from those participants Metacognitively Aware was used as a maximum for a uniform to model $\mathrm{H}_{1}$ in order to test the sensitivity of each participant's Logd1'. Each corresponding $B$ allowed determining their CA as significant (i.e., aware of the stimulus-reward contingencies), sensitively null (i.e., unaware of the contingencies), or insensitive (neither sensitively aware nor unaware). ${ }^{6}$

\footnotetext{
${ }^{6}$ The MatLab script developed to generate this categorization is available online and can be used not only for learning tasks, but also for visual detection experiments: https://osf.io/p7n9b/?view_only=8295819dae61452ebdf4b3d82ccc61 c9.
} 
Table 3. Distribution of participants between contingency awareness and metacognition groups

\begin{tabular}{llcccc}
\hline & & \multicolumn{3}{c}{ Type I awareness } \\
\cline { 3 - 6 } & & Aware & Unaware & Insensitive & Total \\
\hline Type II & Aware & 7 & 0 & 0 & 7 \\
awareness & Unaware & 1 & 11 & 0 & 12 \\
& Insensitive & 8 & 9 & 13 & 30 \\
& Total & 16 & 20 & 13 & 49 \\
\hline
\end{tabular}

Out of the 49 participants who completed the study, on the basis of Type II scores, seven had metacognitive awareness, 12 were definitely Unaware, and 30 showed an insensitive result. On the basis of Type I scores, 16 participants were categorized as being Aware of contingencies, 20 were Unaware, and 13 had an insensitive Bayes factor.

Thus, participants could be categorized as Aware, Unaware, or Insensitive both on a metacognitive and on a contingency awareness level (see Table 3). All analyses reported below were performed on the basis of Type I categorization and only with the 16 Aware and 20 Unaware participants, discarding the 13 participants who showed an Insensitive result.

\section{Questionnaires}

A series of independent samples $t$-tests compared age and scores on questionnaires (BIS, AUDIT, AUQ, PANAS Positive, and BPQ) between Aware and Unaware groups. Due to violations of normality, a Wilcoxon test was performed on PANAS Negative and Reverse Digit Span data. A $\chi^{2}$ was computed to test for differences in gender distribution.

\section{Pleasantness}

A $2 \times 2$ mixed ANOVA with stimulus-type (HR vs. LR) as the within, and CA (Aware vs. Unaware) as the between subjects' factor examined the effects of contingency awareness on pleasantness ratings for HR/LR CS.

A Bayes factor using significant data from Leganes-Fonteneau et al. (2018) as priors was computed for each group separately to quantify differences in pleasantness ratings between HR and LR CS.

\section{$\mathrm{N}$-back task}

One participant was excluded from all analyses involving the $\mathrm{N}$-back task due to low accuracy in the 2-back condition $(<54 \%)$. Given the differences in sample size existing between Aware and Unaware conditions analyses are conducted separately for each group (Leganes-Fonteneau et al. 2018).

\section{Accuracy}

For each of the three accuracy indices we computed a $2 \times 2$ ANOVA with stimulus-type (HR vs. LR) and load (0-back vs. 2-back) as within-subject factors. Due to the observed violations of normality (Shapiro-Wilk tests, $p s<.004$ ) that were nonamendable using transformations, these ANOVAs were performed using ARTool nonparametric analyses for nonnormal distributions in $\mathrm{R}$ (Wobbrock et al. 2011).

\section{Latencies}

Due to violations of normality, all latency scores were log transformed and analyses were performed on the log transformed data. We examined latencies on all trials as well as latencies of target trials only in separate analyses. These analyses used $2 \times 2$ ANOVAs with stimulus-type (HR vs. LR) and load (0-back vs. 2-back) as within-subject factors, and significant interactions were explored using paired-samples $t$-tests.

\section{Flanker task}

Participants with accuracy deviating by more than 2 SDs from the mean in the congruent Control condition were considered to be outliers (Nikolaou et al. 2013b) as their responses in such simple trials (press a key in the direction of all the arrows presented on screen with no distracting stimulus) should not be impaired and may reveal a lack of attention. Consequently 3 participants were excluded from all analyses involving flanker task data. As for the analysis of the $\mathrm{N}$-back task, all analyses described below were computed in each group separately.

\section{Accuracy}

Normality was violated for all accuracy scores. Thus, analyses on accuracy data were performed using ARTool. These used $2 \times 2$ ANOVAs with congruency (congruent vs. incongruent) and stimulus-type (HR vs. LR vs. Control) as within-subject factors. $\chi^{2}$ was performed on interactions followed by Wilcoxon tests.

\section{Latencies}

Latency and flanker effect scores were normally distributed. In order to examine differences in the flanker effect computed for the Control, LR, and HR stimulus-type conditions, we used one-way repeated measures ANOVAs followed by Bonferroni-corrected contrasts for each group.

Analyses of latency data involving $2 \times 2$ ANOVAs, with congruency (congruent vs. incongruent) and stimulus-type (HR vs. LR vs. Control) as within-subject factors are included in the Supplementary Materials. Significant main effects of stimulus-type were followed by post hoc Bonferroni corrected contrasts. Significant stimulus-type by congruency interactions were explored by running one-way repeated measures ANOVAs separately at each level of congruency, and significant effects were followed up further with Bonferroni-corrected contrasts.

\section{Differences between groups}

A set of additional analyses was performed on all the previously described variables for both attentional tasks, including contingency awareness as a between subjects' effect to compare Aware and Unaware groups. In order to overcome differences in sample size and violations of normality a bootstrapping sampling procedure with 1000 iterations was performed across a three-way mixed ANOVA using ezBoot ("ez" package Lawrence and Lawrence 2016). 95\% CIs for the differences between Aware and Unaware participants were obtained across the stimulus by trial type interaction and plotted using ezPlot2. Significant differences are reported in the results section, and the complete results of this analysis are available in the Supplementary Materials.

\section{Acknowledgments}

The authors acknowledge once more Prof. Zoltan Dienes from the University of Sussex for his contribution in the development of Bayesian categorizations of implicit and explicit knowledge.

\section{References}

Anderson BA. 2015. Value-driven attentional capture is modulated by spatial context. Vis $\operatorname{cog} n$ 23: 67-81. doi:10.1080/13506285.2014. 956851

Anderson BA. 2016. What is abnormal about addiction-related attentional biases? Drug Alcohol Depend 167: 8-14. doi:10.1016/j.drugalcdep.2016. 08.002

Anderson BA, Yantis S. 2013. Persistence of value-driven attentional capture. J Exp Psychol Hum Percept Perform 39: 6-9. doi:10.1037/ a0030860

Anderson BA, Laurent PA, Yantis S. 2011. Value-driven attentional capture. Proc Natl Acad Sci 108: 10367-10371. doi:10.1073/pnas.1104047108

Anderson BA, Laurent PA, Yantis S. 2012. Generalization of value-based attentional priority. Vis cogn 20: 647-658. doi:10.1080/13506285.2012. 679711 
Anderson BA, Folk CL, Garrison R, Rogers L. 2016a. Mechanisms of habitual approach: Failure to suppress irrelevant responses evoked by previously reward-associated stimuli. J Exp Psychol Gen 145: 796-805. doi:10.1037/ xge0000169

Anderson BA, Kuwabara H, Wong DF, Gean EG, Rahmim A, Brašić JR, George N, Frolov B, Courtney SM, Yantis S. 2016b. The role of dopamine in value-based attentional orienting. Curr Biol 26: 550-555. doi:10. 1016/j.cub.2015.12.062

Austin AJJ, Duka T. 2010. Mechanisms of attention for appetitive and aversive outcomes in Pavlovian conditioning. Behav Brain Res 213: 1926. doi:10.1016/j.bbr.2010.04.019

Balodis IM, Potenza MN. 2015. Anticipatory reward processing in addicted populations: a focus on the monetary incentive delay task. Biol Psychiatry 77: 434-444. doi:10.1016/j.biopsych.2014.08.020

Barrett AB, Dienes Z, Seth AK. 2013. Measures of metacognition on signal-detection theoretic models. Psychol Methods 18: 535-552. doi:10. $1037 / \mathrm{a} 0033268$

Berridge KC, Robinson TE. 2003. Parsing reward. Trends Neurosci 26: 507513. doi:10.1016/S0166-2236(03)00233-9

Bonson KR, Grant SJ, Contoreggi CS, Links JM, Metcalfe J, Weyl HL, Kurian V, Ernst M, London ED. 2002. Neural systems and cue-induced cocaine craving. Neuropsychopharmacology 26: 376-386. doi:10.1016/ S0893-133X(01)00371-2

Bourgeois A, Neveu R, Vuilleumier P. 2016. How does awareness modulate goal-directed and stimulus-driven shifts of attention triggered by value learning? PLoS One 11: e0160469. doi:10.1371/journal.pone.0160469

Bradley B, Field M, Healy H, Mogg K. 2008. Do the affective properties of smoking-related cues influence attentional and approach biases in cigarette smokers? J Psychopharmacol 22: 737-745. doi:10.1177/ 0269881107083844

Brown CRH, Duka T, Forster S. 2018. Attentional capture by alcohol-related stimuli may be activated involuntarily by top-down search goals. Psychopharmacology (Berl) 235: 2087-2099. doi:10.1007/ s00213-018-4906-8

Carter BL, Tiffany ST. 1999. Meta-analysis of cue-reactivity in addiction research. Addiction 94: 327-340. doi:10.1046/j.1360-0443.1999. 9433273.x

Cox W, Fadardi JS, Pothos EM. 2006. The addiction-stroop test: theoretical considerations and procedural recommendations. Psychol Bull 132: 443. doi:10.1037/0033-2909.132.3.443

De Houwer J. 2006. What are implicit measures and why are we using them. In The handbook of implicit cognition and addiction (ed. Wiers RW, Stacy AW), pp. 11-28. Sage Publishers, Thousand Oaks, CA.

Desimone R, Duncan J. 1995. Neural mechanisms of selective visual attention. Апnи Rev Neurosci 18: 193-222. doi:10.1146/annurev.ne.18. 030195.001205

Dienes Z. 2014. Using Bayes to get the most out of non-significant results. Front Psychol 5: 781. doi:10.3389/fpsyg.2014.00781

Dienes Z. 2015. How Bayesian statistics are needed to determine whether mental states are unconscious. In Behavioural methods in consciousness research (ed. Overgaard M), pp. 199-220. Oxford University Press, Oxford. doi:10.1093/acprof:oso/9780199688890.003.0012

Eriksen CW, Schultz DW. 1979. Information processing in visual search: A continuous flow conception and experimental results. Percept Psychophys 25: 249-263. doi:10.3758/BF03198804

Failing M, Theeuwes J. 2017. Selection history: how reward modulates selectivity of visual attention. Psychon Bull Rev 25: 514-538. doi:10. 3758/s13423-017-1380-y

Field M, Cox WM. 2008. Attentional bias in addictive behaviors: a review of its development, causes, and consequences. Drug Alcohol Depend 97: 120. doi:10.1016/j.drugalcdep.2008.03.030

Field M, Mogg K, Mann B, Bennett GA, Bradley BP. 2013. Attentional biases in abstinent alcoholics and their association with craving. Psychol Addict Behav 27: 71-80. doi:10.1037/a0029626

Garfinkel SN, Seth AK, Barrett AB, Suzuki K, Critchley HD. 2015. Knowing your own heart: distinguishing interoceptive accuracy from interoceptive awareness. Biol Psychol 104: 65-74. doi:10.1016/j. biopsycho.2014.11.004

Garland EL, Franken IHA, Howard MO. 2012. Cue-elicited heart rate variability and attentional bias predict alcohol relapse following treatment. Psychopharmacology (Berl) 222: 17-26. doi:10.1007/ s00213-011-2618-4

Goldstein RZ, Volkow ND. 2002. Drug addiction and its underlying neurobiological basis: neuroimaging evidence for the involvement of the frontal cortex. Am J Psychiatry 159: 1642-1652. doi:10.1176/appi. ajp.159.10.1642

Hester R, Garavan H. 2009. Neural mechanisms underlying drug-related cue distraction in active cocaine users. Pharmacol Biochem Behav 93: 270277. doi:10.1016/j.pbb.2008.12.009

Hogarth L, Dickinson A, Duka T. 2005. Explicit knowledge of stimulus? Outcome contingencies and stimulus control of selective attention and instrumental action in human smoking behaviour. Psychopharmacology (Berl) 177: 428-437. doi:10.1007/s00213-004-1973-9

Hogarth L, Dickinson A, Hutton SB, Bamborough H, Duka T. 2006a. Contingency knowledge is necessary for learned motivated behaviour in humans: relevance for addictive behaviour. Addiction 101: 1153-1166. doi:10.1111/j.1360-0443.2006.01459.x

Hogarth L, Dickinson A, Hutton SB, Elbers N, Duka T. 2006b. Drug expectancy is necessary for stimulus control of human attention, instrumental drug-seeking behaviour and subjective pleasure. Psychopharmacology (Berl) 185: 495-504. doi:10.1007/ s00213-005-0287-x

Jeffs S, Duka T. 2017. Predictive but not emotional value of Pavlovian stimuli leads to Pavlovian-to-instrumental transfer. Behav Brain Res 321: 214222. doi:10.1016/j.bbr.2016.12.022

Kensinger EA, Corkin S. 2003. Effect of negative emotional content on working memory and long-term memory. Emotion 3: 378-393. doi:10. 1037/1528-3542.3.4.378

Krebs RM, Boehler CN, Woldorff MG. 2010. The influence of reward associations on conflict processing in the Stroop task. Cognition 117: 341-347. doi:10.1016/j.cognition.2010.08.018

Kunimoto C, Miller J, Pashler H. 2001. Confidence and accuracy of near-threshold discrimination responses. Conscious Cogn 10: 294-340. doi:10.1006/ccog.2000.0494

Ladouceur CD, Silk JS, Dahl RE, Ostapenko L, Kronhaus DM, Phillips ML. 2009. Fearful faces influence attentional control processes in anxious youth and adults. Emotion 9: 855-864. doi:10.1037/a0017747

Lawrence MA. 2016. Package "ez." ftp://moscoso.org/pub/plan/R/web/ packages/ez/ez.pdf.

LeDoux J. 2002. Cognitive-emotional interactions: Listen to the brain. In Cognitive neuroscience of emotion (ed. Lane RDR, Nadel L, Ahern GL, Allen J, Kaszniak AW), pp. 129-155. Oxford University Press, New York.

Leganes-Fonteneau M, Scott R, Duka T. 2018. Attentional responses to stimuli associated with a reward can occur in the absence of knowledge of their predictive values. Behav Brain Res 341: 26-36. doi:10.1016/j.bbr. 2017.12.015

Le Pelley ME, Pearson D, Griffiths O, Beesley T. 2015. When goals conflict with values: Counterproductive attentional and oculomotor capture by reward-related stimuli. J Exp Psychol Gen 144: 158-171. doi:10.1037/ xge0000037

Le Pelley ME, Seabrooke T, Kennedy BL, Pearson D, Most SB. 2017. Miss it and miss out: Counterproductive nonspatial attentional capture by task-irrelevant, value-related stimuli. Atten Percept Psychophys 79: 16281642. doi:10.3758/s13414-017-1346-1

Lovibond PF, Shanks DR. 2002. The role of awareness in Pavlovian conditioning: empirical evidence and theoretical implications. J Exp Psychol Anim Behav Process 28: 3-26. doi:10.1037/0097-7403.28.1.3

Mehrabian A, Russell JA. 1978. A questionnaire measure of habitual alcohol use. Psychol Rep 43: 803-806. doi:10.2466/pr0.1978.43.3.803

Moeller SJ, Maloney T, Parvaz MA, Dunning JP, Alia-Klein N, Woicik PA, Hajcak G, Telang F, Wang GJ, Volkow ND, et al. 2009. Enhanced choice for viewing cocaine pictures in cocaine addiction. Biol Psychiatry 66: 169-176. doi:10.1016/j.biopsych.2009.02.015

Nikolaou K, Field M, Critchley H, Duka T. 2013a. Acute alcohol effects on attentional bias are mediated by subcortical areas associated with arousal and salience attribution. Neuropsychopharmacology 38: 1365-1373. doi:10.1038/npp.2013.34

Nikolaou K, Field M, Duka T. 2013b. Alcohol-related cues reduce cognitive control in social drinkers. Behav Pharmacol 24: 29-36. doi:10.1097/FBP. Ob013e32835cf458

Patton JH, Stanford MS, Barratt ES. 1995. Factor structure of the Barratt Impulsiveness Scale. J Clin Psychol 51: 768-774. doi:10.1002/1097-4679 (199511)51:6<768::AID-JCLP2270510607>3.0.CO;2-1

Pessoa L, Ungerleider LG. 2004. Neuroimaging studies of attention and the processing of emotion-laden stimuli. Prog Brain Res 144: 171-182. doi:10.1016/S0079-6123(03)14412-3

Pollatos O, Schandry R. 2008. Emotional processing and emotional memory are modulated by interoceptive awareness. Cogn Emot 22: 272-287. doi:10.1080/02699930701357535

Pool E, Sennwald V, Delplanque S, Brosch T, Sander D. 2016. Measuring wanting and liking from animals to humans: a systematic review. Neurosci Biobehav Rev 63: 124-142 doi:10.1016/j.neubiorev.2016.01. 006

Porges S. 1993. Body Perception Questionnaire. Laboratory of Developmental Assessment, University of Maryland, College Park, MD.

Redick TS, Lindsey DRB. 2013. Complex span and n-back measures of working memory: a meta-analysis. Psychon Bull Rev 20: 1102-1113. doi:10.3758/s13423-013-0453-9

Sand A, Nilsson ME. 2016. Subliminal or not? Comparing null-hypothesis and Bayesian methods for testing subliminal priming. Conscious Cogn 44: 29-40. doi:10.1016/j.concog.2016.06.012

San Martín R, Appelbaum LG, Huettel SA, Woldorff MG. 2016. Cortical brain activity reflecting attentional biasing toward reward-predicting 
Implicit reward learning and cognitive control

cues covaries with economic decision-making performance. Cereb Cortex 26: 1-11. doi:10.1093/cercor/bhu160

Saunders JB, Aasland OG, Babor TF, De La Fuente JR, Grant M. 1993.

Development of the Alcohol Use Disorders Identification Test (AUDIT): WHO Collaborative Project on Early Detection of Persons with Harmful Alcohol Consumption-II. Addiction 88: 791-804. doi:10.1111/j. 1360-0443.1993.tb02093.x

Shanks DR. 2016. Regressive research: the pitfalls of post hoc data selection in the study of unconscious mental processes. Psychon Bull Rev 24: 752 775. doi:10.3758/s13423-016-1170-y

Stanislaw H, Todorov N. 1999. Calculation of signal detection theory measures. Behav Res Meth Instrum Comput 31: 137-149. doi:10.3758/ BF03207704

Stewart J, de Wit H, Eikelboom R. 1984. Role of unconditioned and conditioned drug effects in the self-administration of opiates and stimulants. Psychol Rev 91: 251-268. doi:10.1037/0033-295X.91.2.251

Tiffany ST. 1990. A cognitive model of drug urges and drug-use behavior: role of automatic and nonautomatic processes. Psychol Rev 97: 147-168. doi:10.1037/0033-295X.97.2.147

Townshend JM, Duka T. 2001. Attentional bias associated with alcohol cues: differences between heavy and occasional social drinkers. Psychopharmacology (Berl) 157: 67-74. doi:10.1007/s002130100764

Townshend JM, Duka T. 2002. Patterns of alcohol drinking in a population of young social drinkers: a comparison of questionnaire and diary measures. Alcohol Alcohol 37: 187-192. doi:10.1093/alcalc/37.2.187

Uher R, Brooks SJ, Bartholdy S, Tchanturia K, Campbell IC. 2014. Increasing cognitive load reduces interference from masked appetitive and aversive but not neutral stimuli. PLoS One 9: e94417. doi:10.1371/journal.pone. 0094417

Vuilleumier P. 2005. How brains beware: neural mechanisms of emotional attention. Trends Cogn Sci 9: 585-594. doi:10.1016/j.tics.2005.10.011

Watson D, Clark LA, Tellegen A. 1988. Development and validation of brief measures of positive and negative affect: the PANAS scales. J Pers Soc Psychol 54: 1063-1070. doi:10.1037/0022-3514.54.6.1063

Wechsler D. 2008. Wechsler Adult Intelligence Scale-Fourth Edition (WAIS-IV). The Psychological Corporation, San Antonio, TX.

Wiers RW, Stacy AW. 2006. Implicit cognition and addiction. Curr Dir Psychol Sci 15: 292-296. doi:10.1111/j.1467-8721.2006.00455.x

Wiers RW, Stacy AW, Ames SL, Noll JA, Sayette MA, Zack M, Krank M. 2002. Implicit and explicit alcohol-related cognitions. Alcohol Clin Exp Res 26: 129-137. doi:10.1111/j.1530-0277.2002.tb02441.x

Wobbrock JO, Findlater L, Gergle D, Higgins JJ. 2011. The aligned rank transform for nonparametric factorial analyses using only ANOVA procedures. In Proceedings of the ACM Conference on Human Factors in Computing Systems (CHI'11), Vancouver, British Columbia, pp. 143-146, ACM Press, New York. doi:10.1145/1978942.1978963

Yokoyama T, Padmala S, Pessoa L. 2015. Reward learning and negative emotion during rapid attentional competition. Front Psychol 6: 269. doi:10.3389/fpsyg.2015.00269

Received August 22, 2018; accepted in revised form January 11, 2019. 


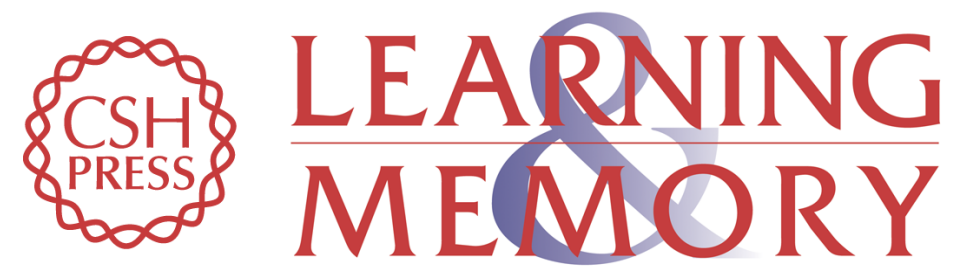

\section{Knowledge about the predictive value of reward conditioned stimuli modulates their interference with cognitive processes}

Mateo Leganes-Fonteneau, Kyriaki Nikolaou, Ryan Scott, et al.

Learn. Mem. 2019, 26:

Access the most recent version at doi:10.1101/Im.048272.118

\section{Supplemental http://learnmem.cshlp.org/content/suppl/2019/02/12/26.3.66.DC1 Material}

References This article cites 65 articles, 1 of which can be accessed free at: http://learnmem.cshlp.org/content/26/3/66.full.html\#ref-list-1

Creative This article is distributed exclusively by Cold Spring Harbor Laboratory Press for the Commons first 12 months after the full-issue publication date (see

License http://learnmem.cshlp.org/site/misc/terms.xhtml). After 12 months, it is available under a Creative Commons License (Attribution-NonCommercial 4.0 International), as described at http://creativecommons.org/licenses/by-nc/4.0/.

Email Alerting Receive free email alerts when new articles cite this article - sign up in the box at the Service top right corner of the article or click here. 